
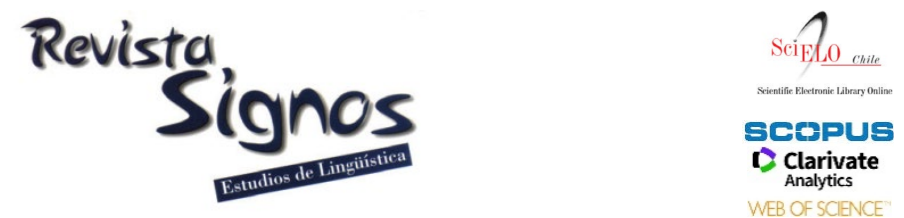

\title{
Formación de nombres en mapudungún: Productividad, genuinidad y planificación*
}

\section{Noun formation in Mapudungun: Productivity, genuineness and language planning}

Belén Villena
Araya
PONTIFICIA UnivERSIDAD CATÓLICA DE
VALPARAíso
ChILE
belen.villena@gmail.com

\author{
María Teresa Cabré \\ Castellví \\ UNIVERSITAT POMPEU FABRA \\ ESPAÑA \\ teresa.cabre@upf.edu
}

\author{
Sabela Fernández- \\ Silva \\ PONTIFICIA UNIVERSIDAD CATÓLICA DE \\ VALPARAÍSO \\ CHILE
}

Recibido: 20-V-2018 / Aceptado: 05-XII-2018

DOI: $10.4067 /$ S0718-09342019000200615

\section{Resumen}

Esta investigación tiene como objetivo principal analizar -desde una perspectiva diacrónica- la productividad de los procedimientos de creación de nombres en mapudungún, partiendo de la hipótesis de que los resultados constatados en periodos de menor contacto con el español exhibirán mayor genuinidad lingüística que los constatados en periodos de un contacto más intenso. Para cumplir este objetivo analizamos los procedimientos de creación de 2.779 nombres, los que clasificamos en tres grupos según su primera fuente de registro: el de registro temprano, compuesto por 274 unidades documentadas en Valdivia (1606); el de registro intermedio, compuesto por 855 unidades documentadas en Febrés ([1765] 1882), y el de registro reciente, compuesto por 1.650 unidades documentadas en Augusta (1916). El análisis de estos tres grupos muestra que ciertos procedimientos mantienen una productividad alta a lo largo del tiempo (composición y derivación), mientras que otros pierden productividad (conversión sintáctica, procedimientos semánticos y préstamos) y otros se mantienen como procedimientos poco productivos (onomatopeya, reduplicación, sintagmación, acortamiento y derivación regresiva). Por otro lado, se constata que, con el paso del tiempo, la creación de nombres en mapudungún pasó de una situación caracterizada por el empleo de diversos procedimientos de productividad alta a otra, que estimamos menos genuina, caracterizada por la concentración de este grado de productividad en unos pocos recursos. Tales resultados podrían servir de base para una política de planificación del léxico que aspire a favorecer la implantación de las nuevas unidades y a fortalecer la identidad lingüística de los hablantes de esta lengua.

Palabras Clave: Formación de nombres, productividad, genuinidad, planificación lingüística, mapudungún. 


\begin{abstract}
The main objective of this research is to analyze -from a diachronic perspective- the productivity of the processes of noun formation in Mapudungun, based on the hypothesis that the processes observed in periods of less contact with Spanish will exhibit greater linguistic genuineness than those found in periods where contact is more intense. For this purpose, we analyzed the formation process of 2,779 nouns, classified into three groups according to their primary source of record: early period, composed of 274 units documented in Valdivia (1606); intermediate period, comprising 855 units documented in Febrés ([1765] 1882); and recent period, which includes 1,650 units documented in Augusta (1916). The analysis reveals that certain processes remain highly productive across the periods (compounding and derivation), others lose productivity (syntactic conversion, semantic changes and borrowing), and others remain unproductive over time (onomatopoeia, reduplication, syntagmatic compounding, clipping and regressive derivation). Furthermore, it is observed that noun formation evolved along time from a situation characterized by a high degree of productivity of several formation processes to another situation in which productivity concentrated on a shorter number of procedures, implying a genuinity loss in our opinion. These results could serve as a basis for the policy planning of a lexicon that aims to encourage the establishment of new units and to strengthen the linguistic identity of the speakers of this language.
\end{abstract}

Key Words: Noun formation, language planning, productivity, genuineness, Mapudungun.

\title{
INTRODUCCIÓN
}

El léxico del mapudungún, al igual que el de la mayor parte de las lenguas indoamericanas, ha sufrido una contracción a lo largo de varios siglos de discriminación cultural y política. Esto ha producido, por un lado, que se pierdan palabras y, por otro, que la lengua no modernice su vocabulario. De esta forma, al entrar en la complejidad del mundo contemporáneo, la lengua mapuche no ha contado ni con unidades léxicas ni con estilos discursivos capaces de expresarla en la diversidad de contextos comunicativos funcionales en los que hoy en día se ven involucrados sus hablantes (Chiodi \& Loncon, 1999).

No obstante esta situación, durante las últimas décadas, la producción verbal escrita en mapudungún ha tenido un importante e inédito desarrollo. Las continuas demandas de diversas organizaciones mapuches, apoyadas por la entrada en vigencia en Chile -el 14 de septiembre de 2009- del Convenio No 169 sobre pueblos indígenas y tribales en países independientes de la Organización Internacional del Trabajo han llevado al mapudungún a ocupar espacios hasta ese momento dominados exclusivamente por el español. Es así como diversos proyectos institucionales en los ámbitos educacional, legal, administrativo y asistencial han propiciado la publicación de abundantes materiales en mapudungún, como por ejemplo leyes, folletos y formularios y señalización de servicios públicos. Esta intensificación de la traducción de textos no tradicionales al mapudungún ha generado la creación de nuevas unidades 
léxicas, frente a lo cual no se ha contado con investigaciones que sirvan de guía para dicha tarea. En este contexto, en el presente trabajo analizamos -desde una perspectiva diacrónica- la productividad de los procedimientos empleados en la creación de nombres en mapudungún. En este análisis, partimos de la hipótesis de que los resultados constatados en periodos de menor contacto con la lengua española exhibirán mayor genuinidad lingüística que los constatados en periodos de un contacto más intenso. El este sentido, entendemos la genuinidad en términos contrastivos y únicamente en relación con la potencial influencia del español (y no de otras lenguas en contacto indígenas) sobre el mapudungún. Finalmente, estos resultados nos servirán de base para inferir criterios que guíen la planificación de neologismos en mapudungún.

\section{Marco teórico: Neología y planificación}

La neología léxica, en tanto disciplina de la lingüística aplicada dedicada al estudio de las palabras nuevas, presenta, al menos, una triple vertiente: lingüística, cultural y política. Dentro de la primera, la labor más importante es la caracterización y tipologización de los neologismos. Dentro de la segunda, la detección de valores y creencias de una comunidad a través de las palabras. Y dentro de la tercera, el establecimiento de criterios que regulen la creación de palabras nuevas y la selección de las formas concurrentes (Cabré, 2002).

Para Cabré (2004), esta última labor puede desarrollarse de diferentes formas, dependiendo del grado de consolidación sociopolítica de las distintas comunidades lingüísticas. En este sentido, mientras más consolidada está una comunidad hablante, más ágil es su capacidad creativa y, dado que los nuevos usos surgen naturalmente por la fuerza social de la lengua, la planificación de neologismos es menos necesaria. En estos casos, la administración asume más bien la función de coordinar y ordenar el proceso de planificación lingüística. En cambio, en las lenguas socialmente más débiles -debido al número de hablantes o a sus condiciones políticas, económicas o socioculturales- la comunidad genera neologismos con mucha prudencia, lo que se manifiesta, por ejemplo, en la vacilación de los hablantes antes de usar neologismos espontáneos en situaciones públicas y formales. A juicio de la autora, tal prudencia creativa se debe, principalmente, a la inseguridad que la utilización de palabras nuevas puede producir en los hablantes por una falta de dominio del sistema lingüístico y de sus registros funcionales. También puede deberse a una censura social frente a posibles transgresiones involuntarias de la norma correcta en el marco de una concepción excesivamente rígida de esta y una uniformidad funcional del sistema de la lengua. En estos casos, la administración debe asumir un rol más activo, generando líneas de investigación e intervención tendientes a generar neologismos que puedan implantarse en la comunidad lingüística. La mayor parte de las lenguas indígenas y, en particular la que es nuestro objeto de estudio, se encuentra en esta última situación. 
Por otro lado, el diseño de una política institucional sobre la neología de una lengua debería priorizar los procesos y recursos más productivos en la creación neológica espontánea, puesto que estos evidenciarían un mayor grado de naturalidad en la creación de palabras, lo que facilitaría su implantación en el uso real de los hablantes (Cabré, Bayà, Bernal, Freixa, Solé \& Vallès, 2002). En este sentido, Cabré (2000: 87) señala que para que la neología logre implantarse en el uso:

"Ha de conèixer els recursos que amb més força $i$ espontaneïtat utilitza la col-lectivitat parlant, analitzar-los, $i$, si els considera convenients, explotar-los al maxim en les seves creacions, per així promoure'ls i confiar a poc a poc la creació de recursos estables a la pròpia comunitat”.

\section{Marco metodológico}

\subsection{Fuentes de extracción de las unidades léxicas}

Los nombres analizados en este trabajo fueron extraídos de dos obras lexicográficas bilingües mapudungún-español: el Diccionario Araucano-Español ó sea Calepino Chileno-Hispano, del jesuita Andrés Febrés, publicado en 1765, y el Diccionario Araucano-Español, del capuchino Félix de Augusta, publicado en 19161. Escogimos estas obras porque han sido las más usadas en el proceso de enseñanza-aprendizaje el mapudungún, tanto en la formación de misioneros (durante los siglos XVII, XVIII, XIX y principios del XX) como en la actualidad.

\subsection{Identificación de las unidades de análisis}

Decidimos que nuestras unidades de análisis fueran exclusivamente sustantivos sobre la base de que, tal como lo han demostrado investigaciones actuales sobre el español y el catalán (Cabré et al., 2002; Fuentes, Gerding, Pecchi, Kotz \& Cañete, 2009), los nombres son los neologismos más productivos, tanto en la neología espontánea como en la planificada.

Ahora bien, los procesos de identificación y extracción de los sustantivos de Augusta (1916) y Febrés ([1765]1882) fueron diferentes en cada uno de ellos: en el primero, las marcas gramaticales asignadas a la gran mayoría de los nombres permitieron orientar estos procesos, mientras que, en el segundo, la escasa presencia de estas marcas nos llevó a considerar la morfología y/o la definición como criterios de selección. De esta forma, obtuvimos un total de 5.113 nombres -2.021 extraídos de Febrés ([1765]1882) y 3.101, de Augusta (1916)-, de los cuales 4.193 correspondieron a formas distintas. De ellas, solo se analizaron desde el punto de vista de su creación 2.779 unidades -un 66\% del total-; el resto no pudo ser analizado por corresponder a nombres monomorfémicos o por presentar una morfología o una semántica opacas. 


\subsection{Constitución de los grupos de análisis}

Para abordar el análisis de los procedimientos de creación de nombres en mapudungún desde una perspectiva diacrónica, establecimos tres grupos de nombres a partir de su primera fuente de registro. En esta tarea, empleamos los repertorios lexicográficos de Febrés ([1765]1882) y Augusta (1916) como fuente de extracción de unidades de análisis, y el Valdivia (1606) como fuente de contraste. El primero de estos grupos -que denominamos grupo de registro temprano (en adelante, RT)corresponde a los nombres documentados por primera vez en Valdivia (1606) -la primera obra lexicográfica del mapudungún-. El segundo -que denominamos grupo de registro intermedio (en adelante, RI) - corresponde a las unidades léxicas documentadas por primera vez en Febrés ([1765]1882). Y el tercero -que denominamos grupo de registro reciente (en adelante, RR)- está compuesto por las unidades documentadas por primera vez en Augusta (1916). En la Tabla 1 se detalla la composición de estos tres grupos.

Tabla 1. Grupos de nombres analizables según su primer registro.

\begin{tabular}{|c|c|c|c|c|c|}
\hline \multirow{2}{*}{ Grupos } & \multirow{2}{*}{ Número total } & \multirow{2}{*}{ Número parcial } & \multicolumn{3}{|c|}{ Fuentes de documentación } \\
\cline { 4 - 6 } & & & Valdivia (1606) & Febrés [1765] & Augusta (1916) \\
\hline RT & \multirow{2}{*}{274} & 110 & $\mathrm{x}$ & $\mathrm{x}$ & $\mathrm{x}$ \\
\cline { 3 - 6 } & & 158 & $\mathrm{x}$ & $\mathrm{x}$ & $\mathrm{x}$ \\
\cline { 3 - 6 } & & 6 & & $\mathrm{x}$ & $\mathrm{x}$ \\
\hline \multirow{2}{*}{ RI } & 855 & 259 & & & $\mathrm{x}$ \\
\hline & & 596 & & & \\
\hline RR & 1.650 & 1.650 & & & \\
\hline
\end{tabular}

\subsection{Modelo de análisis de procedimientos de formación}

Para identificar y describir los procedimientos de creación nominal en mapudungún, utilizamos como base el modelo de clasificación de neologismos propuesto por Cabré (2006), el que adaptamos según los recursos de formación constatados en nuestro corpus, tal como se expone en la Tabla 2: 
Tabla 2. Modelo de clasificación de los procedimientos de formación nominal.

\begin{tabular}{|c|c|c|c|c|}
\hline \multirow{12}{*}{ Formación propia } & \multirow{5}{*}{ Combinación } & Fonológica & \multicolumn{2}{|c|}{ Onomatopeya } \\
\hline & & \multirow{3}{*}{ Morfológica } & \multicolumn{2}{|c|}{ Derivación } \\
\hline & & & \multicolumn{2}{|c|}{ Composición } \\
\hline & & & \multicolumn{2}{|c|}{ Reduplicación } \\
\hline & & Sintáctica & \multicolumn{2}{|c|}{ Sintagmación } \\
\hline & \multirow{5}{*}{ Cambio } & Gramatical & \multicolumn{2}{|c|}{ Conversión sintáctica } \\
\hline & & \multirow{4}{*}{ Semántico } & \multirow{2}{*}{\multicolumn{2}{|c|}{$\begin{array}{l}\text { Restricción } \\
\text { Ampliación }\end{array}$}} \\
\hline & & & & \\
\hline & & & \multirow{2}{*}{ Cambio } & Metáfora \\
\hline & & & & Metonimia \\
\hline & \multirow{2}{*}{ Reducción } & \multicolumn{3}{|c|}{ Acortamiento } \\
\hline & & \multicolumn{3}{|c|}{ Derivación regresiva } \\
\hline \multirow{2}{*}{$\begin{array}{l}\text { Adopción de } \\
\text { préstamos }\end{array}$} & \multirow{2}{*}{ Lengua fuente } & \multicolumn{3}{|c|}{ Adaptados gráficamente } \\
\hline & & \multicolumn{3}{|c|}{ No adaptados gráficamente } \\
\hline
\end{tabular}

\section{Presentación y discusión de los resultados}

\subsection{Análisis de la productividad de procedimientos de creación de nombres}

En el grupo de RT observamos que el procedimiento más productivo en la creación de nombres es la composición, la que se emplea para formar el 28,10\%, seguida de los procedimientos semánticos, con un 20,44\%; la derivación, con un $14,96 \%$; la adopción de préstamos, con un 13,14\%, y la conversión sintáctica, con un $11,31 \%$. El resto de los procedimientos presenta un rendimiento inferior al 10\%: la reduplicación forma el 4,74\% de las unidades; la derivación regresiva, el 4,38\%; la onomatopeya, el 2,55\%, y el acortamiento, el 0,36\%, como se aprecia en la Tabla 3.

Tabla 3. Productividad de los procedimientos de creación en el conjunto de nombres de RT.

\begin{tabular}{|l|c|c|c|c|c|}
\hline \multirow{3}{*}{ Procedimientos específicos } & \multicolumn{5}{|c|}{ RT } \\
\cline { 2 - 6 } & $\mathbf{v - f - a}$ & $\mathbf{v - f}$ & $\mathbf{v - a}$ & \multicolumn{2}{|c|}{ Total } \\
\cline { 2 - 6 } & $\mathbf{n}^{\mathbf{0}}$ & $\mathbf{n}^{\mathbf{0}}$ & $\mathbf{n}^{\mathbf{0}}$ & $\mathbf{n}^{\mathbf{0}}$ & $\mathbf{\%}$ \\
\hline Onomatopeya & 4 & 3 & - & $\mathbf{7}$ & $\mathbf{2 , 5 5}$ \\
\hline Derivación & 17 & 23 & 1 & $\mathbf{4 1}$ & $\mathbf{1 4 , 9 6}$ \\
\hline Composición & 19 & 56 & 2 & $\mathbf{7 7}$ & $\mathbf{2 8 , 1 0}$ \\
\hline Reduplicación & 8 & 4 & 1 & $\mathbf{1 3}$ & $\mathbf{4 , 7 4}$ \\
\hline Sintagmación & - & - & - & - & - \\
\hline Conversión sintáctica & 11 & 18 & 2 & $\mathbf{3 1}$ & $\mathbf{1 1 , 3 1}$ \\
\hline Procedimientos semánticos & 26 & 30 & - & $\mathbf{5 6}$ & $\mathbf{2 0 , 4 4}$ \\
\hline Acortamiento & 1 & - & - & $\mathbf{1}$ & $\mathbf{0 , 3 6}$ \\
\hline Derivación regresiva & 5 & 7 & - & $\mathbf{1 2}$ & $\mathbf{4 , 3 8}$ \\
\hline Préstamos & 19 & 17 & - & $\mathbf{3 6}$ & $\mathbf{1 3 , 1 4}$ \\
\hline Total & $\mathbf{1 1 0}$ & $\mathbf{1 5 8}$ & $\mathbf{6}$ & $\mathbf{2 7 4}$ & $\mathbf{1 0 0 , 0 0}$ \\
\hline
\end{tabular}

Nota. Las abreviaturas corresponden a v: Valdivia (1606); f: Febrés ([1765]1882); a: Augusta (1916). 
En el grupo de RI, en tanto, observamos que el procedimiento más productivo en la creación de nombres sigue siendo la composición, la que se emplea para formar el 39,65\%; seguida de los procedimientos semánticos, con un $14,27 \%$; la conversión sintáctica, con un 13,92\%, y la derivación, con un 10,76\%. El resto de los procedimientos presenta un rendimiento inferior al 10\%: la adopción de préstamos crea el 6,55\% de las unidades; la reduplicación y la derivación regresiva, el 5,38\%; el acortamiento, el 3,04\%; la onomatopeya, el 0,82\%, y la sintagmación, el 0,23\%, como se aprecia en la Tabla 4.

Tabla 4. Productividad de los procedimientos de creación en el conjunto de nombres de RI.

\begin{tabular}{|l|c|c|c|c|}
\hline \multirow{2}{*}{ Procedimientos específicos } & \multicolumn{4}{|c|}{ RI } \\
\cline { 2 - 5 } & $\mathbf{f - a}$ & $\mathbf{f}$ & \multicolumn{2}{|c|}{ Total } \\
\cline { 2 - 5 } & $\mathbf{n}^{\mathbf{0}}$ & $\mathbf{n}^{\mathbf{o}}$ & $\mathbf{n}^{\mathbf{0}}$ & $\mathbf{0}$ \\
\hline Onomatopeya & 7 & - & $\mathbf{7}$ & $\mathbf{0 , 8 2}$ \\
\hline Derivación & 32 & 60 & $\mathbf{9 2}$ & $\mathbf{1 0 , 7 6}$ \\
\hline Composición & 99 & 240 & $\mathbf{3 3 9}$ & $\mathbf{3 9 , 6 5}$ \\
\hline Reduplicación & 24 & 22 & $\mathbf{4 6}$ & $\mathbf{5 , 3 8}$ \\
\hline Sintagmación & - & 2 & $\mathbf{2}$ & $\mathbf{0 , 2 3}$ \\
\hline Conversión sintáctica & 34 & 85 & $\mathbf{1 1 9}$ & $\mathbf{1 3 , 9 2}$ \\
\hline Procedimientos semánticos & 20 & 102 & $\mathbf{1 2 2}$ & $\mathbf{1 4 , 2 7}$ \\
\hline Acortamiento & 4 & 22 & $\mathbf{2 6}$ & $\mathbf{3 , 0 4}$ \\
\hline Derivación regresiva & 10 & 36 & $\mathbf{4 6}$ & $\mathbf{5 , 3 8}$ \\
\hline Préstamos & 29 & 27 & $\mathbf{5 6}$ & $\mathbf{6 , 5 5}$ \\
\hline Total & $\mathbf{2 5 9}$ & $\mathbf{5 9 6}$ & $\mathbf{8 5 5}$ & $\mathbf{1 0 0 , 0 0}$ \\
\hline
\end{tabular}

Por último, en el grupo de RR observamos que el procedimiento más productivo es -al igual que en los otros grupos- la composición, la que se emplea para formar el $49,45 \%$ de las unidades; seguida de la derivación, con un 17,58\%. El resto de los procedimientos presenta un rendimiento inferior al 10\%: la conversión sintáctica crea el 8,24\% de las unidades; los procedimientos semánticos, el 6,85\%; la derivación regresiva, el 5,21\%; la reduplicación, el 4,79\%; la adopción de préstamos, el 3,82\%, la onomatopeya, el 1,27\%, y la sintagmación, el 0,42\%, como se aprecia en la Tabla 5.

Tabla 5. Productividad de los procedimientos de creación en el conjunto de nombres de RR.

\begin{tabular}{|l|r|r|}
\hline Procedimientos específicos & \multicolumn{1}{c|}{$\mathbf{n}^{\mathbf{0}}$} & \multicolumn{1}{c|}{$\mathbf{0}$} \\
\hline Onomatopeya & 21 & 1,27 \\
\hline Derivación & 290 & 17,58 \\
\hline Composición & 816 & 49,45 \\
\hline Reduplicación & 79 & 4,79 \\
\hline Sintagmación & 7 & 0,42 \\
\hline Conversión sintáctica & 136 & 8,24 \\
\hline Procedimientos semánticos & 113 & 6,85 \\
\hline Acortamiento & 39 & 2,36 \\
\hline Derivación regresiva & 86 & 5,21 \\
\hline Préstamos & 63 & 3,82 \\
\hline Total & $\mathbf{1 6 5 0}$ & $\mathbf{1 0 0 , 0 0}$ \\
\hline
\end{tabular}


Si comparamos la productividad de los procedimientos de creación nominal en los tres grupos, observamos que, en ellos, el más productivo es la composición, mediante la cual se forma el $28,10 \%$ de las unidades de RT, el 39,65\% de las de RI y el 49,45\% de las de RR. El segundo procedimiento en productividad es, en los primeros dos grupos, el de tipo semántico, con un $20,44 \%$ en el primero y un $14,27 \%$ en el segundo; en el último grupo, en cambio, el segundo lugar lo ocupa la derivación, con un $17,58 \%$. El tercer procedimiento en rendimiento es, en el primer grupo, la derivación, con un $14,96 \%$, y en el segundo y tercero, la conversión sintáctica, con un $13,92 \%$, y un $8,24 \%$, respectivamente. El cuarto procedimiento en productividad es, en el primer grupo, la adopción de préstamos, con un 13,14\%; en el segundo, la derivación, con un 10,76\%, y en el tercero, los procedimientos semánticos, con un 6,91\%. El resto de los procedimientos (con excepción de la conversión en el grupo de RT) exhibió una productividad baja, inferior al 10\%, como se expone en la Tabla 6 .

Tabla 6. Comparación de la productividad de los procedimientos de creación en los tres grupos.

\begin{tabular}{|l|r|r|r|r|r|r|}
\hline \multirow{2}{*}{ Procedimientos específicos } & \multicolumn{6}{|c|}{ Grupos } \\
\cline { 2 - 7 } & \multicolumn{2}{|c|}{$\mathbf{R T}$} & \multicolumn{2}{|c|}{ RI } & \multicolumn{2}{c|}{ RR } \\
\cline { 2 - 7 } & $\mathbf{n}^{\mathbf{0}}$ & \multicolumn{1}{c}{$\mathbf{\%}$} & \multicolumn{1}{c|}{$\mathbf{n}^{\mathbf{0}}$} & \multicolumn{1}{c}{$\mathbf{0}$} & \multicolumn{1}{c|}{$\mathbf{n}^{\mathbf{0}}$} & \multicolumn{1}{c}{$\mathbf{0}$} \\
\hline Onomatopeya & 7 & 2,55 & 7 & 0,82 & 21 & 1,27 \\
\hline Derivación & 41 & 14,96 & 92 & 10,76 & 290 & 17,58 \\
\hline Composición & 77 & 28,10 & 339 & 39,65 & 816 & 49,45 \\
\hline Reduplicación & 13 & 4,74 & 46 & 5,38 & 79 & 4,79 \\
\hline Sintagmación & - & - & 2 & 0,23 & 7 & 0,42 \\
\hline Conversión sintáctica & 31 & 11,31 & 119 & 13,92 & 136 & 8,24 \\
\hline Procedimientos semánticos & 56 & 20,44 & 122 & 14,27 & 113 & 6,85 \\
\hline Acortamiento & 1 & 0,36 & 26 & 3,04 & 39 & 2,36 \\
\hline Derivación regresiva & 12 & 4,38 & 46 & 5,38 & 86 & 5,21 \\
\hline Préstamos & 36 & 13,14 & 56 & 6,55 & 63 & 3,82 \\
\hline Total & $\mathbf{2 7 4}$ & $\mathbf{1 0 0 , 0 0}$ & $\mathbf{8 5 5}$ & $\mathbf{1 0 0 , 0 0}$ & $\mathbf{1 6 5 0}$ & $\mathbf{1 0 0}$ \\
\hline
\end{tabular}

Por otro lado, en la Figura 1 podemos ver los cambios que ha experimentado la productividad de cada procedimiento a lo largo del tiempo: aumenta en los casos de la composición y la derivación; disminuye en los casos de la conversión, los procedimientos semánticos y los préstamos; y se mantiene baja (no supera el 5\%) en los caso de la onomatopeya, la reduplicación, la sintagmación, el acortamiento y la derivación regresiva. De todos estos cambios, los más significativos son el aumento exhibido por la composición y la disminución experimentada por los procedimientos semánticos y la adopción de préstamos. 


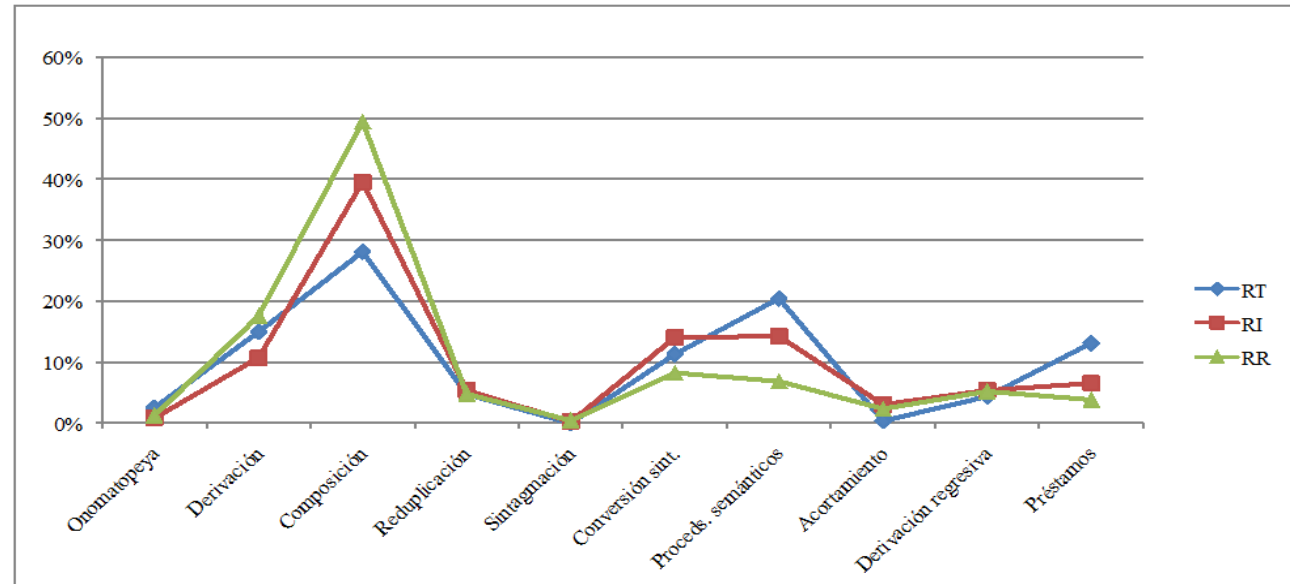

Figura 1. Comparación de la productividad de los procedimientos de creación en los tres grupos.

\subsection{Análisis estadístico}

Para determinar la relación entre las variables, aplicamos la prueba estadística bilateral chi cuadrado $\left(\chi^{2}\right)$ de Pearson, con un nivel de confianza de 95\%. Para identificar las categorías de las variables en las que podría hallarse una asociación, analizamos los valores de los residuos estandarizados obtenidos mediante la prueba anterior (hay significancia si los valores de los residuos son mayores que 1,96 o menores que $-1,96)$.

Los resultados del test chi cuadrado nos permiten establecer que el periodo de registro de las unidades (RT, RI, RR) incide en la productividad de los distintos procedimientos de creación de nombres $\left(\chi^{2}(16, \mathrm{~N}=2779)=174,842, p<0,05\right)$. Con respecto a estos últimos, el análisis de los residuos estandarizados, expuestos en la Tabla 7, revela que los recursos asociados al tiempo son: los semánticos, la composición, la adopción de préstamos, la conversión sintáctica y la derivación. Los valores residuales muestran que la representación de estos procedimientos es similar en los grupos de RT y RI, pero diferente en el de RR. Los procedimientos semánticos, la conversión sintáctica y la adopción de préstamos están sobrerrepresentados en los primeros dos grupos e infrarrepresentados en el último, mientras que la composición y la derivación exhiben la situación contraria: están infrarrepresentados en los primeros dos grupos y sobrerrepresentados en el último². 
Tabla 7. Valores de los residuos estandarizados para los procedimientos de creación nominal.

\begin{tabular}{|l|c|c|c|}
\hline \multirow{2}{*}{ Procedimientos específicos } & \multicolumn{3}{|c|}{ Grupos } \\
\cline { 2 - 4 } & RT & RI & RR \\
\hline Onomatopeya & 1,9 & $-1,2$ & 0,1 \\
\hline Derivación & $-0,1$ & $-3,4$ & 2,5 \\
\hline Composición & $-4,1$ & $-2,1$ & 3,2 \\
\hline Reduplicación & $-0,2$ & 0,5 & $-0,3$ \\
\hline Conversión sintáctica & 0,5 & 3,4 & $-2,7$ \\
\hline Procedimientos semánticos & 5,0 & 3,4 & $-4,5$ \\
\hline Acortamiento & $-2,2$ & 1,3 & 0,0 \\
\hline Derivación regresiva & $-0,6$ & 0,2 & 0,1 \\
\hline Préstamos & 5.3 & 1,2 & $-3,0$ \\
\hline
\end{tabular}

\subsection{Descripción de los procedimientos de creación de nombres}

\subsubsection{Onomatopeya}

La onomatopeya, procedimiento mediante el que se crean "unidades léxicas cuyo significado está relacionado con las propiedades acústicas del significante" (Alcaraz \& Martínez, 1997: 392), presenta una productividad muy baja. En los tres grupos se emplea casi exclusivamente para crear zoónimos y, dentro de ellos, nombres de aves. Este resultado coincide con lo planteado por los estudios anteriores que destacan su importancia en la formación de términos de la zoología, especialmente de la ornitología (Villagrán, Villa, Hinojosa, Sánchez, Romo, Maldonado, Cavieres, Latorre, Cuevas, Castro, Papic \& Valenzuela, 1999; Navarro, 2014). En el grupo de RT, los 7 nombres formados mediante este procedimiento corresponden a nombres de aves que imitan su canto, como (1a). En el grupo de RI, los 7 nombres formados mediante este recurso corresponden a zoónimos: 6, a nombres de aves, como (1b) y, uno, a un insecto (1c); todos estos nombres imitan el canto de los animales que denominan. En el grupo de RR, 20 de los 21 nombres creados mediante este proceso corresponden a zoónimos: 15, a nombres de aves, como (1d); 3, a anfibios, como (1e); y 2, a insectos, como (1f); la mayor parte de ellos imita el canto de los animales que denominan. El único nombre fuera del ámbito de la zoología se presenta en $(1 \mathrm{~g})$.

(1) a. chili 'un pajarito negro, como tordo [...]

b. chukaw 'un pájaro del monte'

c. dille 'una chicharra'

d. püchiw 'el pájaro pichíu o pitigüe. Colaptes Pitius'

e. ngakiñ 'cierto sapito. Los indígenas dicen que tiene una voz fina y alta, como de perro nuevo, y la remedan con nga nga nga'

f. ñufnuf 'cierto insecto del tamaño y forma de una hormiga [...]. Tiene su nombre de su modo de andar en la superficie de la tierra como olfateando'

g. chüli 'el retintín, el sonido que producen algunos cuerpos sonoros al chocar con otros' 


\subsubsection{Derivación}

En el ámbito de la creación de nombres en mapudungún, cuando hablamos de derivación nos referimos exclusivamente a la sufijación, entendida como la adjunción de un sufijo a una base léxica ${ }^{3}$. En el conjunto de nombres formados mediante este procedimiento se constata la presencia de diversos sufijos, algunos de los cuales están presentes en los tres grupos, mientras que otros exhiben un uso discontinuo. Los primeros sufijos mencionados son -we (instrumental), -we (locativo), -fe, -peyüm (instrumental), -wen y -ïll, los que crean casi la totalidad de los nombres derivados: el $95,12 \%$ del primer grupo, el $78,27 \%$ del segundo y el $80,34 \%$ del tercero, como se expone en la Tabla 8.

Tabla 8. Productividad de los sufijos de uso continuo.

\begin{tabular}{|l|c|c|c|c|c|c|}
\hline \multirow{2}{*}{ Sufijos } & \multicolumn{6}{|c|}{ Grupos } \\
\cline { 2 - 7 } & \multicolumn{2}{|c|}{ RT } & \multicolumn{2}{|c|}{ RI } & \multicolumn{2}{c|}{ RR } \\
\cline { 2 - 7 } & $\mathbf{n}^{\mathbf{0}}$ & $\mathbf{\%}$ & $\mathbf{n}^{\mathbf{0}}$ & $\mathbf{\%}$ & $\mathbf{n}^{\mathbf{0}}$ & $\mathbf{\%}$ \\
\hline -we (inst) & 23 & 56,10 & 41 & 44,57 & 67 & 23,10 \\
\hline -we (loc) & 6 & 14,63 & 19 & 20,65 & 25 & 8,62 \\
\hline -fe & 7 & 17,07 & 7 & 7,61 & 110 & 37,93 \\
\hline -wen & 2 & 4,88 & 4 & 4,35 & 29 & 10,00 \\
\hline - ïll & 1 & 2,44 & 1 & 1,09 & 2 & 0,69 \\
\hline Total & $\mathbf{3 9}$ & $\mathbf{9 5 , 1 2}$ & $\mathbf{7 2}$ & $\mathbf{7 8 , 2 7}$ & $\mathbf{2 3 3}$ & $\mathbf{8 0 , 3 4}$ \\
\hline
\end{tabular}

De ellos, los más productivos son el instrumental y el locativo -we, como en (2a-b), y el agentivo $-f e$, como en (2c). El cuarto sufijo en productividad es el pluralizador asociativo -wen, como en (2d), y, el quinto, el apreciativo - ïll. Este último es un afijo que no ha sido descrito en la literatura revisada y cuya existencia planteamos a partir de la evidencia analizada en esta investigación. Este sufijo -fosilizado en la etapa actual de la lengua pero posiblemente más productivo en etapas anteriores- se añadiría a bases nominales para darles un sentido apreciativo diminutivo, como en ( $2 \mathrm{f}-\mathrm{g})$.
a. lepü-we
(lepü- 'borrar')
'la escoba'
b. chadi-we
(chadi- 'haber sal')
'las salinas donde van por sal'
c. deyka-ve
(deyka- 'sajar')
'sajador'
d. peñi-wen
(peñi 'hermano de ego
'ser dos hermanos o ser de un
masculino')
e. chang-ïll
(chang 'rama')
f. vod-üll
(fodï 'espina')
g. pangk-ïll
(pangi 'el puma')
mismo padre o vientre'
'ramito'
'el huesito de la fruta'
'cachorro del puma'

En cuanto a los sufijos de uso discontinuo, estos se presentan en la Tabla 9. 
Tabla 9. Productividad de los sufijos de uso discontinuo.

\begin{tabular}{|l|l|l|l|l|l|l|}
\hline \multirow{2}{*}{ Sufijos } & \multicolumn{6}{|c|}{ Grupos } \\
\cline { 2 - 7 } & \multicolumn{2}{|c|}{ RT } & \multicolumn{2}{c|}{ RI } & \multicolumn{2}{c|}{ RR } \\
\cline { 2 - 7 } & $\mathbf{n}^{\mathbf{o}}$ & $\mathbf{\%}$ & $\mathbf{n}^{\mathbf{0}}$ & $\mathbf{0}$ & $\mathbf{n}^{\mathbf{o}}$ & $\mathbf{\%}$ \\
\hline -keyüm (loc) & 1 & 2 & 1 & 1 & - & - \\
\hline -keyüm (inst) & - & - & 4 & 5 & - & - \\
\hline -peyüm (inst) & 1 & 2 & - & - & 17 & 6 \\
\hline -peyüm (loc) & - & - & 1 & 1 & 8 & 3 \\
\hline -(e)ntu & - & - & 6 & 7 & 16 & 5 \\
\hline -en & - & - & 2 & 3 & 5 & 2 \\
\hline -l & - & - & 5 & 5 & 8 & 3 \\
\hline -(y)em & - & - & - & - & 3 & 1 \\
\hline Total & $\mathbf{2}$ & $\mathbf{4}$ & $\mathbf{1 9}$ & $\mathbf{2 2}$ & $\mathbf{5 7}$ & $\mathbf{2 0}$ \\
\hline
\end{tabular}

Los sufijos locativo e instrumental -keyüm -variantes morfológicas de -peyümforman nombres como (3a-b). Estos sufijos nominalizadores se originan a partir de la lexicalización de las secuencias de sufijos verbales -ke-y-üm y -pe-y-üm, respectivamente, donde -ke- es un marcador de rasgo constante; - $p e^{-}$, marcador de proximidad; $-y$-, fonema epentético, y - üm, forma no finita que crea nombres verbales instrumentales. En los grupos de nombres de RT y RI podemos ver cómo ambas secuencias, en vías de lexicalización, coexisten y compiten entre sí, lexicalizándose e imponiéndose el uso de la segunda de ellas, puesto que es la que se mantienen en el grupo de RR. Junto con ellos, observamos el empleo discontinuo de los sufijos locativo e instrumental -peyüm, como en (3c-d), del colectivizador -(e)ntu, como en (3e), del pacientivo -en, como en (3f), del marcador de obsolescencia -(y)em, como en (3g) y del imitativo -l, como en (3h-j). Este último sufijo ha sido escasamente descrito en la literatura revisada; solo lo menciona Smeets (2008), quien no profundiza en su análisis. En esta investigación planteamos, a partir de la evidencia analizada, que este sufijo -fosilizado en la etapa actual de la lengua pero posiblemente más productivo en etapas anteriores- se agregaría a bases nominales para formar nombres que denotan el mismo significado de la base, pero de un modo imperfecto, imitativo, una 'especie de' el significado de la base.

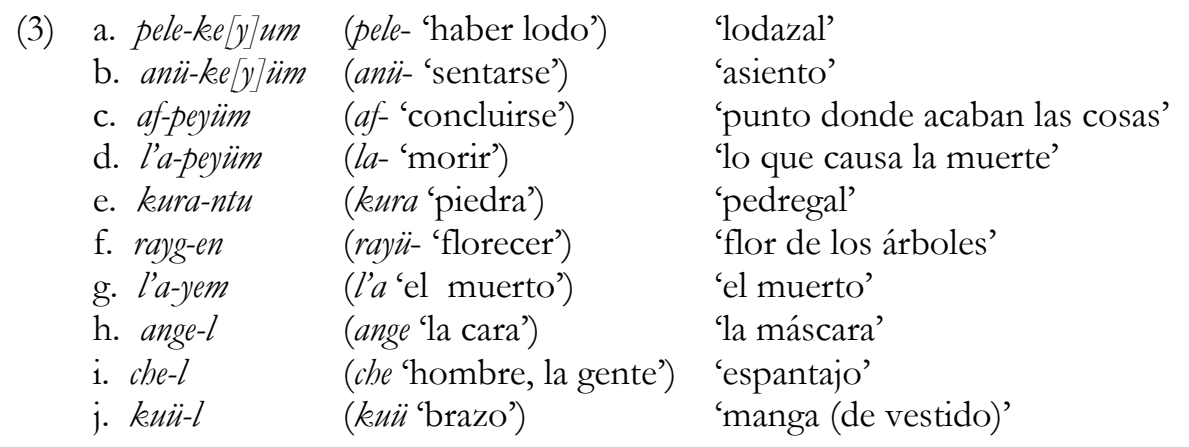




\subsubsection{Composición}

La composición, recurso que consiste en la unión de dos o más bases léxicas, es sumamente productiva en los tres grupos de registro considerados: forma 77 nombres en el primero, 339, en el segundo, y 816, en el tercero. Como se expone en la Tabla 10, los compuestos N-N, como (4a), son los más numerosos en los tres grupos; en segundo lugar se encuentran los compuestos $\operatorname{Adj}-\mathrm{N}$, como (4b); en tercer lugar están los compuestos V-N, como (4c); y en cuarto y quinto lugar se encuentran los compuestos Adv-N, como (4d) y Prep-N, como (4e), los que exhiben una productividad muy baja.

Tabla 10. Tipos de compuestos según la categoría gramatical de sus constituyentes.

\begin{tabular}{|l|c|c|c|c|c|c|}
\hline \multirow{2}{*}{ Tipos de compuestos } & \multicolumn{6}{|c|}{ Grupos } \\
\cline { 2 - 7 } & \multicolumn{2}{|c|}{ RT } & \multicolumn{2}{c|}{ RI } & \multicolumn{2}{c|}{ RR } \\
\cline { 2 - 7 } & $\mathbf{n}$ & $\mathbf{0}$ & $\mathbf{n}$ & $\mathbf{\%}$ & $\mathbf{n}$ & $\mathbf{\%}$ \\
\hline N-N & 40 & 51,95 & 188 & 55,46 & 510 & 62,50 \\
\hline Adj-N & 18 & 23,38 & 73 & 21,53 & 129 & 15,81 \\
\hline V-N & 13 & 16,88 & 49 & 14,46 & 89 & 10,91 \\
\hline Adv-N & 1 & 1,30 & 10 & 2,95 & 6 & 0,74 \\
\hline Prep-N & 1 & 1,30 & - & - & 3 & 0,37 \\
\hline Indet. & 4 & 5,19 & 19 & 5,60 & 79 & 9,68 \\
\hline Total & $\mathbf{7 7}$ & $\mathbf{1 0 0 , 0 0}$ & $\mathbf{3 3 9}$ & $\mathbf{1 0 0 , 0 0}$ & $\mathbf{8 1 6}$ & $\mathbf{1 0 0 , 0 0}$ \\
\hline
\end{tabular}

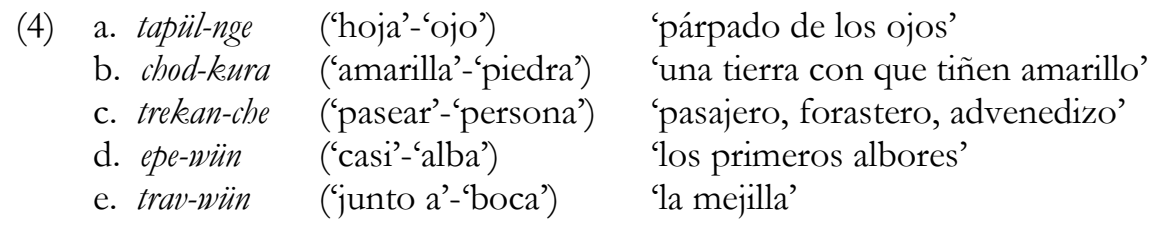

\subsubsection{Reduplicación}

En el conjunto de nombres creados mediante reduplicación, es decir, mediante la repetición de material fonológico, podemos encontrar, por una parte, casos de reduplicación total, como (5a); y de reduplicación parcial, como (5b).
a. fida-fida 'el paladar duro' (RR)
b. tro-l-tro 'cerraja'

En todos los grupos de registro, el primer tipo supera ampliamente al segundo, como se aprecia en la Tabla 11.

Tabla 11. Tipos de reduplicación según grupos de registro.

\begin{tabular}{|c|c|c|c|}
\hline \multirow{2}{*}{ Tipos } & \multicolumn{3}{|c|}{ Grupos } \\
\cline { 2 - 4 } & RT & RI & RR \\
\hline Total & 9 & 41 & 68 \\
\hline Parcial & 4 & 5 & 11 \\
\hline Total & $\mathbf{1 3}$ & $\mathbf{4 6}$ & $\mathbf{7 9}$ \\
\hline
\end{tabular}




\subsubsection{Sintagmación}

La sintagmación o lexicalización de estructuras sintácticas es, en nuestro corpus, un proceso muy poco productivo y muy limitado temática y estructuralmente. En el grupo de RI, los dos nombres creados mediante este procedimiento forman parte del campo temático del medio ambiente (específicamente, de los cuerpos celestes) y su estructura es V-N, como (6a). En el grupo de RR, de los 7 nombres creados mediante este proceso, 5 forman parte del léxico de las tradiciones religiosas. De ellos, 4 presentan la estructura V-N, como (6b); y uno, la estructura Prep-N-V (6c). De los 2 restantes, uno pertenece al ámbito doméstico y presenta nuevamente la estructura V-N (6d) y otro forma parte del ámbito de la flora y presenta la estructura N-Pos-N (6e).
a. lay-antï
b. antüpay-ñamko
('murió el sol')
'eclipse de sol'
('vino del sol el
'águila venida del sol' águila')
c. pu-pülli-amuy
('se fue al interior
'cierto arte de curación de del suelo') las machis'
d. ̈̈yyümngekey-kütral
('era encendido el
'vela'
fuego')
e. pillu-ñi-weke
('vellón de la garza')
'la hierba sietevenas'

\subsubsection{Conversión sintáctica}

Dentro del conjunto de nombres creados mediante conversión sintáctica o cambio de categoría gramatical de una unidad sin modificación de la base léxica, -31, en el grupo de RT; 119, en el de RI, y 136, en el de RR-, constatamos la presencia de verbos y adjetivos que se convierten en nombres. Como se aprecia en la Tabla 12, en los tres grupos de registro casi la totalidad de las unidades se forma mediante la nominalización de formas verbales.

Tabla 12. Categoría gramatical de las unidades convertidas en nombres según grupos de registro.

\begin{tabular}{|c|c|c|c|}
\hline \multirow{2}{*}{ Categoría gramatical } & \multicolumn{3}{|c|}{ Grupos } \\
\cline { 2 - 4 } & RT & RI & RR \\
\hline Verbos & 31 & 112 & 128 \\
\hline Adjetivos & - & 7 & 8 \\
\hline Total & $\mathbf{3 1}$ & $\mathbf{1 1 9}$ & $\mathbf{1 3 6}$ \\
\hline
\end{tabular}

Con respecto a la conversión de adjetivos, este tipo de conversión, muy poco productiva, se observa solo en los dos últimos grupos, en nombres como ( $7 \mathrm{a}-\mathrm{b})$.
b. kelï ('colorado')
'el cardenal'
c. awka ('alzado, rebelde, muy travieso')
'la yegua' 
En cuanto a la conversión de verbos, todos los casos registrados corresponden a formas no personales del verbo; específicamente, a formas en -(ï)n, -lu, -el, -m y-(ü)wma, como se aprecia en la Tabla 13.

Tabla 13. Formas no finitas convertidas en nombres según grupos de registro.

\begin{tabular}{|c|c|c|c|}
\hline \multirow{2}{*}{ Formas no finitas } & \multicolumn{3}{|c|}{ Grupos } \\
\cline { 2 - 4 } & RT & RI & RR \\
\hline$-(\ddot{u}) n$ & 28 & 108 & 100 \\
\hline$-l u$ & 2 & 2 & 12 \\
\hline$-e l$ & 1 & 2 & 14 \\
\hline$-m$ & - & - & 1 \\
\hline$-(\ddot{u}) w m a$ & - & - & 1 \\
\hline Total & $\mathbf{3 1}$ & $\mathbf{1 1 2}$ & $\mathbf{1 2 8}$ \\
\hline
\end{tabular}

En esta misma Tabla, se observa que, de ellos, las formas en -(ü)n, como (8a), son las más productivas en los tres grupos. El resto de formas no finitas presenta una productividad muy inferior. Las formas en $-l u$, como (8b), se registran en los tres grupos; al igual que las formas en -el, como (8c). Las formas restantes, en - $m$ y en -(ü)wma, se registran solo una vez en el último grupo (8d-e).
a. eltu-n
('enterrar')
b. katrüpe-lu
('que siega')
'su sepultura'
c. ya-el
('que ha de ser comido')
'segador'
'comida'
d. fülkonmo-m
('donde se entra en
'la familia del o la consorte' contacto')
e. l'antu-wma
('que ha enviudado y se casó de nuevo’)
'[la] que ha enviudado y se
casó de nuevo'

Estos resultados confirman, en los tres grupos de registro, los hallazgos de Fernández Garay (2006), quien constata, en la variedad ranquelina del mapudungún, que de las formas verbales no finitas, la forma en -(ï)n es la que exhibe menos determinaciones verbales y más nominales, lo que evidencia su alejamiento de la clase verbal para acercarse más a la nominal. Por tanto, esta forma no finita, concluye la autora, corresponde al sustantivo verbal por excelencia, es decir, es la más nominalizada del sistema.

\subsubsection{Procedimientos semánticos}

En el conjunto de nombres creados mediante procedimientos semánticos -56 en el grupo de RT; 120, en el de RI, y 113, en el de RR- constatamos la presencia de nombres originados a partir de metáforas, como (9a-b), metonimias, como (9c-d), o de la ampliación o reducción del significado de una base léxica ${ }^{4}$, como (9e-f).
a. changüll
('ramito')
b. rawilma
('choroy')
c. waka
('vaca')

'parte de razonamiento'

'fig. la gente locuaz'

'ganado'
(R'T)

(RR) 
d. chillka ('carta') 'papel'

e. trarive ('un cinchón de cuero de 'cualquier otro cinchón

vaca que traen atado a la para amarrar o atadura'

(RT) cintura')

f. pel'echaru ('vaso de greda')

'el crisol de los plateros, que también es de greda'

Con respecto a la productividad de cada procedimiento semántico en particular, vemos que la metáfora y la metonimia son los más productivos en los tres grupos, mientras que la ampliación o reducción semántica exhibe una productividad menor; la metáfora es el recurso con mayor rendimiento en los grupos de RT y RR y la metonimia lo es en el grupo de RI. La metáfora se mantiene en los tres grupos como un procedimiento altamente productivo (en cada grupo origina más de la mitad de los nombres creados mediante procedimientos semánticos), mientras que la metonimia pierde progresivamente productividad, a la vez que la ampliación o reducción semántica aumentan su rendimiento, como se desprende de la Tabla 14.

Tabla 14. Comparación de la productividad de los procedimientos semánticos en los tres grupos.

\begin{tabular}{|c|c|c|c|c|c|c|}
\hline \multirow{2}{*}{ Procedimientos semánticos } & \multicolumn{6}{|c|}{ Grupos } \\
\cline { 2 - 8 } & \multicolumn{2}{|c|}{$\mathbf{R T}$} & \multicolumn{2}{|c|}{ RI } & \multicolumn{2}{c|}{ RR } \\
\cline { 2 - 8 } & $\mathbf{n}^{\mathbf{0}}$ & $\mathbf{\%}$ & $\mathbf{n}^{\mathbf{0}}$ & $\mathbf{\%}$ & $\mathbf{n}^{\mathbf{0}}$ & $\mathbf{\%}$ \\
\hline Metáfora & 29 & 51,79 & 54 & 44,26 & 61 & 53,98 \\
\hline Metonimia & 26 & 46,43 & 63 & 51,64 & 35 & 30,97 \\
\hline Ampliación o reducción & 1 & 1,79 & 4 & 3,28 & 15 & 13,27 \\
\hline Indet. & - & - & 1 & 0,82 & 2 & 1,77 \\
\hline Total & $\mathbf{5 6}$ & $\mathbf{1 0 0 , 0 0}$ & $\mathbf{1 2 2}$ & $\mathbf{1 0 0 , 0 0}$ & $\mathbf{1 1 3}$ & $\mathbf{1 0 0 , 0 0}$ \\
\hline
\end{tabular}

\subsubsection{Acortamiento}

La creación de nombres mediante el acortamiento o reducción de una unidad léxica es un recurso poco productivo: solo hemos encontrado un caso en el grupo de RT; 26, en el grupo de RI, y 39, en el grupo de RR. Los nombres creados mediante este procedimiento pueden ser analizados considerando el proceso de formación de las unidades más extensas de las cuales son su reducción. Así, constatamos que estos nombres pueden surgir a partir del acortamiento de unidades compuestas, como (10a); reduplicadas, como (10b), o derivadas, como (10c).
a. kadi (kadi-voro 'hueso del costado')
b. piroy (piroy-piroy)
c. dungul (dungul-ve 'el que hace hablar')
'costilla, costillar'
'la peonza'
'dicen al lliwa o adivino, porque hace hablar al diablo, aunque las más veces todo es ficción' 


\subsubsection{Derivación regresiva}

La derivación regresiva es aquella por la cual se aplica un proceso de sustracción al lexema base, de lo que se obtiene una unidad derivada que es más simple desde el punto de vista morfológico que el lexema del que se originó (Varela, 2005). Este procedimiento no ha sido descrito sistemáticamente en mapudungún, aunque sí encontramos menciones en algunas gramáticas (Febrés, 1765; Augusta, 1903; Moesbach, 1962; Smeets, 2008). Augusta, por ejemplo, menciona la existencia de adjetivos acabados en -pe-ye, sufijos verbales de proximidad y de temporal de característica constante, respectivamente, que pueden ser usados como sustantivos, como i-pe-ye 'comestible' y werkü-pe-ye 'mandadero' (Augusta, 1903). También indica la posibilidad de formar sustantivos a partir de la raíz verbal künu- 'dejar, poner', como ñido-künu 'el jefe de un trabajo', y a partir del sufijo verbal -kantu que, agregado a bases nominales, expresa la idea de la base, pero de un modo imperfecto e imitativo, como püñen-kantu 'muñeca'. Estos sufijos finales de palabra no son ni nominales ni nominalizadores, sino, más bien, verbales, por lo que postulamos la existencia de formas verbales morfológicamente más complejas, de las cuales las unidades anteriormente mencionadas fueron derivadas regresivamente.

Ahora bien, en cuanto a la forma verbal morfológicamente más compleja que serviría de base para los nombres creados mediante derivación regresiva, Augusta (1916) entrega cierta información relevante. En el primer tomo de su diccionario, en la tercera definición del lema künu señala que este es un "apócope de künuel (el que ha sido hecho algo o lo que uno ha sido hecho) en sustantivos como chelkünu espantajo, ñidokünu jefe de un trabajo, kenputukünu hombre hecho cuñado" (Augusta, 1916: 86); en el mismo sentido, señala que püñmoyenye "mujer que había sido nuera de cierto hombre, hombre que había sido suegro de cierta mujer y que ya no lo son por haberse efectuado un divorcio' es un apócope del participio pasivo pünmoyenyeel (Augusta, 1916). En el segundo tomo de su diccionario, en tanto, en el artículo lexicográfico de la raíz larg-, entrega el ejemplo petu al'üley rüpü ñi inaya 'todavía es largo el camino que he de seguir', del que señala que la palabra inaya 'que he de seguir' es apócope de inayagel o de inayam (Augusta, 1916).

Esta información se ve reforzada por el hallazgo de Smeets (2008) de los siguientes ejemplos de participios pasivos marcados por tiempo pluscuamperfecto -(ï)uye: inche $\tilde{n i}$ wünetu trafie-nyye wenüy fewla lay 'el amigo mío a quien me había encontrado el otro día ahora está muerto' e iñche ñi angkad-unye iñche ñi wenüy 'el que he tomado en la parte posterior [de mi caballo] [es] un amigo mío'. Para estos casos, la autora plantea tentativamente el morfema cero como alternante del sufijo -el después de -(ii)mye para indicar el participio pasivo. Toda esta evidencia sugiere que las formas más complejas que sirven de base a los procesos de derivación regresiva de nombres podrían ser participios pasivos en función verbal o nominal. Sin embargo, es indudable que son 
necesarias más investigaciones que ayuden a dilucidar este y otros puntos del proceso de derivación regresiva.

Ahora bien, los nombres creados mediante este procedimiento -12, en el grupo de RT; 46, en el de RI, y 86, en el de RR- pueden ser clasificados, según sus características formales, en dos grupos: los que están compuestos por dos bases (nominales y/o verbales) y los que presentan una base seguida de uno o más sufijos verbales. El primer grupo lo conforman 58 unidades, de las cuales 38 presentan la estructura V-N; 11, la estructura N-V, y 10, la estructura V-V, como se expone en la Tabla 15.

Tabla 15. Estructuras de las bases presentes en los nombres formados por derivación regresiva.

\begin{tabular}{|c|c|c|c|}
\hline \multirow{2}{*}{ Estructura de la base } & \multicolumn{3}{|c|}{ Grupos } \\
\cline { 2 - 4 } & RT & RI & RR \\
\hline V-N & 5 & 13 & 20 \\
\hline N-V & - & 1 & 10 \\
\hline V-V & - & 5 & 5 \\
\hline Total & $\mathbf{5}$ & $\mathbf{1 9}$ & $\mathbf{3 5}$ \\
\hline
\end{tabular}

Como se desprende de la Tabla 15, la estructura más frecuente en los tres grupos de registro es la compuesta por una base verbal y un elemento nominal incorporado a su derecha, como (11a). La segunda estructura en rendimiento -presente únicamente en los dos últimos grupos- es la compuesta por dos bases verbales, como (11b). En último lugar, la estructura menos frecuente $-\mathrm{y}$ al igual que la anterior, presente solo en los dos últimos grupos- es la compuesta por una base verbal y un elemento nominal incorporado a su izquierda, como (11c).
a. trari-küw ('atar'-'mano')
b. chong-tüku
('apagarse'-'poner')
'manilla o esposa de las manos'
c. ad-entu
('el exterior'-'sacar')
'el tizón'

(RR)

El segundo grupo, en tanto, lo conforman 85 nombres en los que se constata la presencia de una base y uno o más sufijos verbales. En estas unidades apreciamos la presencia de un total de 16 sufijos verbales finales: el continuativo -ka, el imitativo -kantu, el marcador de característica constante -ke, -këntu $u^{5}$, los causativos -(ü)l y -(ï) $m$, el andativo -me, el aplicativo -(ü)ñma, el cislocativo -pa, el indicador de proximidad - $p$ e, el indicador de evento sin objetivo -püra, el verbalizador o aplicativo -tu, el recíproco o reflexivo $-(u) w$ y el marcador de característica constante -ye. En (12) entregamos ejemplos de nombres que presentan estos afijos.
a. trem-üm-ka (trem- 'crecer, criarse')
b. che-l-kantu (che 'persona')
'(hijo) de crianza'
c. mange-l
'el espantajo'
(mange- 'ir a bebidas')
'convidado a la bebida (o comida)' 


\begin{tabular}{|c|c|c|}
\hline d. anü-m & (anü- 'sentarse') & $\begin{array}{l}\text { 'en combinación con vasijas: su } \\
\text { asiento' }\end{array}$ \\
\hline e. küpa-l-me & (küpa- 'venir') & 'descendencia, familia' \\
\hline f. $r e w-[\tilde{n}] m a$ & (rew- 'ola del río') & 'ola del río’' \\
\hline g. el-pa & (el- ‘poner o dejar') & 'descendencia' \\
\hline h. la-n-pe & $(l a-'$ 'morir') & 'la viuda' \\
\hline i. vucha-p[ü]ra & (vucha- 'viejo') & $\begin{array}{l}\text { 'soltero, que envejeció así sin } \\
\text { casarse o de balde' }\end{array}$ \\
\hline j. koñi-n-tu & $\begin{array}{l}\text { (koñi 'hijo, hija de la } \\
\text { mujer') }\end{array}$ & $\begin{array}{l}\text { 'entenados y sobrinos de la tía } \\
\text { materna' }\end{array}$ \\
\hline k. i-lel-ka-w & $(i-$ 'comer') & 'comida de cada uno o su porción' \\
\hline m. i-pe-ye & $(i-$ 'comer') & 'el manjar' \\
\hline n. wekëu-pe-ye & (werk:̈̈-'enviar') & 'mandadero' \\
\hline
\end{tabular}

\subsubsection{Préstamos}

Los nombres creados mediante la adopción de préstamos -36, en el grupo de RT; 56, en el de RI, y 63, en el de RR- pueden ser analizados desde distintos puntos de vista, como la lengua de la cual provienen, el grado de adaptación que presentan y el campo temático al cual pertenecen. Desde el primer punto de vista, constatamos la presencia de 46 préstamos directos del quechua y 109 préstamos directos del español. Como se aprecia en la Tabla 16, en el grupo de nombres de RT los quechuismos son mucho más numerosos que los hispanismos, con una diferencia de 66,66\% en favor de los primeros; mientras que en los grupos de unidades de RI y RR la tendencia es la contraria: los hispanismos superan a los quechuismos en un 53,58\% y 90,48\%, respectivamente. De esta manera, constatamos la paulatina, pero sostenida e importante pérdida de productividad de los préstamos del quechua a lo largo del tiempo.

Tabla 16. Comparación de la productividad de los préstamos según su lengua fuente.

\begin{tabular}{|c|c|c|c|c|c|c|}
\hline \multirow{2}{*}{ Préstamos según lengua fuente } & \multicolumn{6}{|c|}{ Grupos } \\
\cline { 2 - 7 } & \multicolumn{2}{|c|}{ RT } & \multicolumn{2}{|c|}{ RI } & \multicolumn{2}{c|}{ RR } \\
\cline { 2 - 7 } & $\mathbf{n}^{\mathbf{0}}$ & $\mathbf{\%}$ & $\mathbf{n}^{\mathbf{0}}$ & $\mathbf{\%}$ & $\mathbf{n}^{\mathbf{0}}$ & $\mathbf{\%}$ \\
\hline Español & 6 & 16,67 & 43 & 76,79 & 60 & 95,24 \\
\hline Quechua & 30 & 83,33 & 13 & 23,21 & 3 & 4,76 \\
\hline Total & $\mathbf{3 6}$ & $\mathbf{1 0 0 , 0 0}$ & $\mathbf{5 6}$ & $\mathbf{1 0 0 , 0 0}$ & $\mathbf{6 3}$ & $\mathbf{1 0 0 , 0 0}$ \\
\hline
\end{tabular}

En cuanto a los quechuismos, todos se presentan adaptados ortográficamente. En cuanto a los campos temáticos, estos se utilizan fundamentalmente para denominar entidades pertenecientes al ámbito doméstico (los alimentos, la casa y la vestimenta), como (13a), y a las tradiciones (lúdicas, sociales y religiosas), como (13b). 

a. amchi (hamchi 'afrecho, salvado')
b. marikun (kamarikuy 'obsequiar, hacer presentes para conseguir protección')

'afrecho'
'ngillatun, rogativa
solemne'

Respecto de los hispanismos, la mayoría de ellos se presenta con adaptación ortográfica; solo dos unidades (14a-b) no presentan dicha adaptación. Con respecto a los campos temáticos, los hispanismos se utilizan fundamentalmente para referirse a las actividades productivas (agricultura, comercio, ganadería, orfebrería, pesca, tejido y otras), como (14c), al ámbito doméstico (los alimentos, la casa y la vestimenta), como (14d) y a las tradiciones (bélicas, ecuestres, lúdicas, musicales, religiosas y sociales), como (14e).
a. cucbillo
(cucbillo)
'cuchillo'
b. esclavo
(esclavo)
'esclavo'
(RR)
c. akucha
(aguja)
'aguja'
d. charawilla
(zaragüelles)
'el calzón'
e. foshal
(bozal)
'(del castell.) el bozal'

\section{CONCLUSIONES}

En esta investigación nos propusimos analizar -desde una perspectiva diacrónicala productividad de los procedimientos utilizados en la creación de nombres en mapudungún para -a partir de este análisis- inferir criterios que guíen la planificación de neologismos en esta lengua. Los resultados obtenidos nos permiten establecer tres tipos de procedimientos según el grado de productividad exhibido: máximo, alto (más de $10 \%$ de rendimiento) y bajo (menos de $10 \%$ ). El primer tipo de procedimiento fue el mismo en los tres grupos: la composición; sin embargo, los otros dos tipos se organizaron de manera diferente en los tres grupos. En el primero, cuatro procedimientos exhibieron una productividad alta: los semánticos, la derivación, la adopción de préstamos y la conversión sintáctica; y cinco, una productividad baja: la reduplicación, la derivación regresiva, la onomatopeya, el acortamiento y la sintagmación (sin productividad). En el segundo grupo, la adopción de préstamos disminuyó su rendimiento, por lo que los procedimientos de productividad baja aumentaron de cinco a seis. En el tercero, en tanto, los procedimientos semánticos y la conversión sintáctica experimentaron la misma evolución, por lo que los procedimientos de productividad baja aumentaron de seis a ocho. Estos cambios en el grado de productividad de los procedimientos provocaron que, en el último grupo, solo un procedimiento, la derivación, presentara un grado de productividad alto. De este modo, vemos que, con el tiempo, algunos procedimientos aumentaron su rendimiento (la composición y la derivación), mientras que otros perdieron productividad (los procedimientos semánticos, los préstamos y la conversión sintáctica) y otros no sufrieron cambios importantes (la derivación regresiva, la reduplicación, la onomatopeya, el acortamiento y la sintagmación). 
De este último análisis podemos concluir que, con el paso del tiempo, la creación de nombres en mapudungún pasó de una situación caracterizada por el empleo de diversos procedimientos de productividad alta a otra caracterizada por la concentración de este grado de productividad en unos pocos recursos. De esta manera, la situación inicial de los grupos de RT y RR, caracterizada por la presencia de una variedad de procedimientos de productividad alta (cuatro y tres, los que crearon el $59,85 \%$ y el 39,06\% de los nombres, respectivamente), cambió en el grupo de RR, en el que solo un procedimiento exhibió este grado de productividad (el que formó el $17,5 \%$ de los nombres). Por tanto, estimamos que en la creación de nombres en mapudungún es mucho más genuino utilizar diversos procedimientos de manera productiva que concentrar la productividad en unos pocos. De acuerdo con esto, una política de planificación de nombres en mapudungún que aspire a una mayor genuinidad lingüística, debería fomentar la utilización de dos tipos de procedimientos: los que implican un cambio (semántico o sintáctico) y la adopción de préstamos. Estos recursos exhibieron una productividad alta en el primer grupo de registro, menor en el segundo, y baja en el tercero, disminución que creemos que podría deberse a la influencia del español. Tal influencia podría haber originado, también, el aumento de productividad de la derivación, si consideramos que este recurso ha sido el más productivo en la formación de neologismos léxicos en el español de Chile (Fuentes et al., 2009).

De esta forma, sostenemos que, en el contexto actual de revaloración del mapudungún como símbolo de identidad étnica que ha venido acompañada de un profuso e inédito desarrollo de la producción verbal en esta lengua, la planificación de nuevos nombres debería, por un lado, promover el uso de la composición -el procedimiento más productivo en los tres grupos de nombres analizados- y la derivación -recurso con una productividad ascendente que fue el segundo en rendimiento en el último grupo-, puesto que la utilización de los procedimientos más productivos podría favorecer la implantación real en el uso de las nuevas unidades. Y, por otro, debería fomentar el uso de los procedimientos semánticos, la conversión sintáctica y la adopción de préstamos -recursos poco productivos en la actualidad, pero con mayor rendimiento en etapas anteriores de la lengua en las que el contacto con el español era menos intenso-, puesto que su empleo podría fortalecer la identidad lingüística de los hablantes del mapudungún.

\section{REFERENCIAS BIBLIOGRÁFICAS}

Alcaraz, E. \& Martínez, M. A. (1997). Diccionario de lingüística moderna. Barcelona: Ariel.

Augusta, F. de. (1903). Gramática araucana. Valdivia: Imprenta Central, J. Lampert.

Augusta, F. de (1916). Diccionario Araucano-Español y Español-Araucano. Tomo Primero y Tomo Segundo. Santiago: Imprenta Universitaria. 
Cabré, M. T. (2000). La neologia com a mesura de la vitalitat interna de les llengües. La neologia en el tombant de segle. I Simposi sobre neologia (18 de desembre de 1998). I Seminari de neologia (17 de febrer del 2000) (85-108). Barcelona: Institut Universitari de Lingüística Aplicada. Universitat Pompeu Fabra.

Cabré, M. T. (2002). La neologia avui: El naixement d'una disciplina. En M. T. Cabré, E. Solé \& J. Freixa (Coords.), Lèxic $i$ neologia (pp. 29-42). Barcelona: Observatori de Neologia. Institut Universitari de Lingüística Aplicada. Universitat Pompeu Fabra.

Cabré, M. T. (2004). La importància de la neologia per al desenvolupament sostenible de la llengua catalana. En J. Freixa \& E. Solé (Coords.), Llengua catalana y neologia (pp. 17-45). Barcelona: Observatori de Neologia. Institut Universitari de Lingüística Aplicada. Universitat Pompeu Fabra.

Cabré, M. T. (2006). La clasificación de neologismos: Una tarea compleja. Alfa, 50(2), $229-250$.

Cabré, M. T., Bayà, M. R., Bernal, E., Freixa, J., Solé, E. \& Vallès, T. (2002). Evaluación de la vitalidad de una lengua a través de la neología: A propósito de la neología espontánea y de la neología planificada. En M. T. Cabré, E. Solé \& Freixa J. (Coords.), Lèxic i neologia (pp. 159-201). Barcelona: Observatori de Neologia. Institut Universitari de Lingüística Aplicada. Universitat Pompeu Fabra.

Chiodi, F. \& Loncon, E. (1999). Crear nuevas palabras. Innovación y expansión de los recursos lexicales del mapuzugun. Santiago de Chile: Universidad de la Frontera y Corporación Nacional de Desarrollo Indígena.

Febrés, A. (1765). Arte de la Lengua General del Reyno de Chile, con un dialogo ChilenoHispano muy curioso: A que se añade la Doctrina Christiana, esto es, Rezo, Catecismo, Coplas, Confesionario, y Pláticas, lo más en lengua chilena y castellana: Y por fin un vocabulario hispano-chileno, y un calepino chileno-hispano más copioso. Lima, en la Calle de la Encarnación [en línea]. Disponible en: http://www.memoriachilena.cl/602/w3-article-8486.html

Febrés, A. ([1765] 1882). Diccionario Araucano-Español ó sea Calepino Chileno-Hispano Por el P. Andrés Febrés de la Compañia de Jesus. Reproducido textualmente de la edición de Lima de 1765, por Juan M. Larsen. Con un Apéndice sobre las lenguas Quíchua, Aimará y Pampa. Buenos Aires: Juan A. Alsina.

Fernández Garay, A. (2006). Entre el sustantivo y el verbo. Las nominalizaciones del mapuche ranquelino. LIAMES, 6, 61-75. 
Fuentes, M., Gerding, C., Pecchi, A,. Kotz, G. \& Cañete P. (2009). Neología léxica: Reflejo de la vitalidad del español de Chile. Revista de Lingüistica Teórica y Aplicada, 47(1), 103-124 [en línea]. Disponible en: http://www.scielo.cl/pdf/rla/v47n1/art_06.pdf

Moesbach, E. de. (1962). Idioma mapuche. Padre Las Casas: Editorial San Francisco.

Navarro, H. (2014). Vocabulario etnobiológico en mapudungun. En M. Malvestitti \& P. Dreidemie (Comps), III Encuentro de Lenguas Indigenas Americanas (ELIA). Libro de actas (pp. 417-429). Río Negro: Universidad Nacional.

Salas, A. (1992). El mapuche o araucano. Fonología, gramática y antología de cuentos. Madrid: MAPFRE.

Smeets, I. (2008). A grammar of Mapuche. Berlín, Nueva York: Mouton de Gruyter.

Valdivia, L. de (1606). Arte y gramatica general de la Lengva que corre en todo el Reyno de Chile, con un Vocabulario, y Confessonario. Compuestos por el Padre Luys de Valdivia de la Compañia de Iesus en la Prouincia del Piru [en línea]. Disponible en: http://www.cervantesvirtual.com/obra/arte-y-gramatica-general-de-lalengua-que-corre-en-todo-el-reyno-de-chile-con-vn-vocabulario-yconfessonario-compuestos-por-el-padre-luys-de-valdiuia---iuntamente-con-ladoctrina-christiana-y-cathecismo-del-concilio-de-lima-en-espanol-y-dostraduciones-del-en-la-lengua-de-chile-que-examinaron-y-aprobaron-los-dosreuerendissimos-senores-de-chile-cada-qual-la-de-su-obispado/

Varela, S. (2005). Morfología léxica: La formación de palabras. Madrid: Gredos.

Villagrán, C., Villa, R., Hinojosa, L., Sánchez, G., Romo, M., Maldonado, A., Cavieres, L., Latorre, C., Cuevas, J., Castro, S., Papic, C. \& Valenzuela, A. (1999). Etnozoología Mapuche: Un estudio preliminar. Revista Chilena de Historia Natural, 72, 595-627.

Villena, B., Chandía, A., Araya, I. \& Flores, C. (2018). Corpus lexicográfico del mapudungún (CORLEXIM) [en línea]. Disponible en: http:// corlexim.cl

\section{NOTAS}

${ }^{1}$ Estas obras fueron consultadas a través del Corpus Lexicográfico del Mapudungún (CORLEXIM).

${ }^{2}$ La sintagmación no pudo ser procesada por presentar muy pocos casos.

${ }^{3}$ La prefijación en mapudungún es escasa y se desarrolla en el plano verbal (Salas, 1992: 178). 
${ }^{4}$ En el análisis de los nombres creados mediante procedimientos semánticos no fue posible determinar en todos los casos el significado que sirvió de base para los procesos de ampliación, reducción o cambio; en ellos solo identificamos los tipos de relaciones establecidas entre los nombres (metafóricas o metonímicas) sin identificar su dirección y, en los casos de ampliación y reducción semánticas, tratamos en una misma categoría los nombres creados mediante estos procesos.

${ }^{5}$ Desconocemos el significado de este afijo. Tal vez se trate de una variante de -kantu.

\section{AGRADECIMIENTOS}

* Esta investigación ha sido financiada por las becas de doctorado del Programa BECASCHILE, convocatoria 2010, del Fondo Nacional de Fomento del Libro y la Lectura, convocatoria 2016, y por la beca postdoctoral DI/2018 de la Pontificia Universidad Católica de Valparaíso. Agradecemos la colaboración de Fernando Zúñiga durante todo el desarrollo de este trabajo. 